九州大学学術情報リポジトリ

Kyushu University Institutional Repository

Development of mice without Cip/Kip CDK inhibitors

\title{
立石，悠基
}

https://doi.org/10.15017/1500571

出版情報：九州大学，2014，博士（医学），課程博士 バージョン:

権利関係：やむを得ない事由により本文ファイル非公開（2） 


\section{Development of mice without Cip/Kip CDK inhibitors}

Yuki Tateishi, ${ }^{\mathrm{a}, \mathrm{b}}$ Akinobu Matsumoto, ${ }^{\mathrm{a}, \mathrm{b}}$ Tomoharu Kanie, ${ }^{\mathrm{a}, \mathrm{b}}$ Eiji Hara, ${ }^{\mathrm{c}}$ Keiko Nakayama, ${ }^{\mathrm{d}}$ and Keiichi I. Nakayama ${ }^{\mathrm{a}, \mathrm{b}, *}$

${ }^{\mathrm{a}}$ Department of Molecular and Cellular Biology, Medical Institute of Bioregulation, Kyushu University, 3-1-1 Maidashi, Higashi-ku, Fukuoka, Fukuoka 812-8582, Japan

${ }^{\mathrm{b}}$ CREST, Japan Science and Technology Agency, 4-1-8 Honcho, Kawaguchi, Saitama 3320012, Japan

${ }^{\mathrm{c}}$ Cancer Institute, Japanese Foundation for Cancer Research, 3-8-31 Ariake, Koto-ku, Tokyo 135-8550, Japan

${ }^{\mathrm{d}}$ Department of Developmental Genetics, Center for Translational and Advanced Animal Research, Graduate School of Medicine, Tohoku University, 2-1 Seiryo-machi, Aoba-ku, Sendai, Miyagi 980-8575, Japan

*Corresponding author 


\begin{abstract}
Timely exit of cells from the cell cycle is essential for proper cell differentiation during embryogenesis. Cyclin-dependent kinase (CDK) inhibitors (CKIs) of the Cip/Kip family (p21, p27, and p57) are negative regulators of cell cycle progression and are thought to be essential for development. However, the extent of functional redundancy among Cip/Kip family members has remained largely unknown. We have now generated mice that lack all three Cip/Kip CKIs (TKO mice) and compared them with those lacking each possible pair of these proteins (DKO mice). We found that the TKO embryos develop normally until midgestation but die around embryonic day (E) 13.5, slightly earlier than p27/p57 DKO embryos. The TKO embryos manifested morphological abnormalities as well as increased rates of cell proliferation and apoptosis in the placenta and lens that were essentially indistinguishable from those of p27/p57 DKO mice. Unexpectedly, the proliferation rate and cell cycle profile of mouse embryonic fibroblasts (MEFs) lacking all three Cip/Kip CKIs did not differ substantially from those of control MEFs. The abundance and kinase activity of CDK2 were markedly increased, whereas CDK4 activity and cyclin D1 abundance were decreased, in both p27/p57 DKO and TKO MEFs during progression from $\mathrm{G}_{0}$ to $\mathrm{S}$ phase compared with those in control MEFs. The extents of the increase in CDK2 activity and the decrease in CDK4 activity and cyclin D1 abundance were greater in TKO MEFs than in p27/p57 DKO MEFs. These results suggest that p27 and p57 play an essential role in mouse development after midgestation, and that p21 plays only an auxiliary role in normal development (although it is thought to be a key player in the response to DNA damage).
\end{abstract}

Keywords: cell cycle; cyclin-dependent kinase inhibitor (CKI); Cip/Kip CKI family; p21; p27; p57 


\section{Introduction}

Cell proliferation is thought to be controlled by the balance between cyclindependent kinases $(\mathrm{CDKs})^{1}$ and their inhibitors (CKIs). In general, CDKs promote cell cycle progression, whereas CKIs function to restrain it. CKIs bind to various cyclin-CDK complexes and thereby inhibit their kinase activities. They are categorized in two families on the basis of their structure and specificity for different cyclin-CDK complexes [1]. Members of the Ink4 family- $16^{\operatorname{Ink} 4 a}(\mathrm{p} 16), \mathrm{p} 15^{\operatorname{Ink} 4 b}(\mathrm{p} 15), \mathrm{p} 18^{\operatorname{Ink} 4 c}(\mathrm{p} 18)$, and $\mathrm{p} 19^{\operatorname{Ink} 4 d}(\mathrm{p} 19)$ - are inhibitors specific for CDK4 or CDK6, whereas those of the Cip/Kip family, including $\mathrm{p} 21^{\text {Cip1 }}(\mathrm{p} 21), \mathrm{p} 27^{\text {Kip } 1}(\mathrm{p} 27)$, and $\mathrm{p} 57^{\text {Kip2 }}$ (p57), mainly target CDK2 and CDK4 or CDK6 (and CDK1 in some situations) for inhibition. In addition, all Cip/Kip CKIs promote the association of CDK4 with D-type cyclins [2].

Members of the Cip/Kip family share a conserved $\mathrm{NH}_{2}$-terminal domain that contributes to association with and inhibition of cyclin-CDK complexes [1,3]. However, the remaining portions of the three proteins do not share sequence similarity, suggesting that Cip/Kip CKIs may have additional distinct functions or regulatory mechanisms. For example, transcription of the p21 gene is activated in response to expression of p53 induced by DNA damage, resulting in cell cycle arrest in $G_{1}$ or $G_{2}$ phases of the cell cycle. In contrast, expression of p27 is increased in mitogen-deprived cells or otherwise quiescent cells, and the protein is rapidly exported from the nucleus and degraded as cells enter the cell cycle. Unlike p21 and p27, p57 plays a specific role in embryonic development as well as in maintenance of stem cells in adults [4]. The p57 gene is an imprinted gene, with the maternal allele being preferentially expressed [5].

The function of CKIs has been investigated by the generation of mice lacking each member of the Cip/Kip family. Mice deficient in p21 (p21 KO mice) develop normally and do not show a propensity for the development of spontaneous malignancies up to 1 year of age [6]. Mice lacking p27 (p27 KO mice) have a larger body size compared with control mice, manifest marked organomegaly of the thymus, pituitary gland, and gonads (ovary and testis), and often develop pituitary tumors [7-9]. However, neither p21 KO nor p27 KO mice exhibit gross developmental defects during embryogenesis. In contrast, most mice lacking p57 (p57 KO mice) manifest multiple developmental abnormalities such as cleft palate and defective 
bone formation, resulting in severe dyspnea, dysphagia, and eventual death immediately after birth [10-12]. Furthermore, mice lacking all possible pairs of the three Cip/Kip family members have been generated. Whereas mice deficient in both p21 and p27 (p21/p27 DKO mice) survive until adulthood [13, 14], those lacking p21 and p57 (p21/p57 DKO mice) die between embryonic day (E) 16.5 and postnatal day 0 as a result of a placental defect [15]. Mice lacking both p27 and p57 (p27/p57 DKO mice) exhibit the most severe developmental defects. They die in utero between E12 and E16.5 as a result of a developmental abnormality of the placenta and show hypoplasia of many organs in association with an increase in apoptosis [16]. Given that Cip/Kip CKIs have been thought to be essential for embryonic development, it was unexpected that mice with only a single Cip/Kip CKI could develop until E16.5 or adulthood. A possible explanation for such survival in mice with only a single Cip/Kip CKI is that this remaining protein might compensate for the lack of the other two CKIs. To test this possibility, we have now generated and characterized mice lacking all three Cip/Kip CKIs (TKO mice). 


\section{Materials and Methods}

\subsection{Mice}

p21 KO mice were provided by Dr. P. Leder [6]. Generation of p27 KO mice and p57 KO mice was described previously $[9,10]$. All mouse experiments were approved by the Animal Ethics Committee of Kyushu University.

\subsection{Cell culture and flow cytometric analysis}

Primary mouse embryonic fibroblasts (MEFs) were prepared from E13.5 embryos and cultured as previously described [9]. For analysis of synchronized cells, confluent MEFs were rendered quiescent by culture for $48 \mathrm{~h}$ in Dulbecco's modified Eagle's medium supplemented with $0.1 \%$ fetal bovine serum. For analysis of asynchronous cells, MEFs $(1 \times$ $10^{6}$ ) plated in 10-cm culture dishes were cultured for $24 \mathrm{~h}$. Flow cytometric analysis was performed as described [4].

\subsection{RT-PCR, immunoblot analysis, and in vitro CDK activity assay}

Total RNA extracted from cells by the guanidinium thiocyanate-phenolchloroform method with the use of Isogen (Nippon Gene, Tokyo, Japan) was purified and then subjected $(1 \mu \mathrm{g})$ to reverse transcription (RT) with random hexanucleotide primers and reverse transcriptase (Qiagen, Tokyo, Japan). The resulting cDNA was then subjected to polymerase chain reaction (PCR) analysis as described [4]. Immunoblot analysis was performed as described [17]. In vitro CDK activity assay was performed as described [18].

\subsection{Histological analysis}

Embryos were fixed with $4 \%$ paraformaldehyde in phosphate-buffered saline, embedded in paraffin, sectioned with a Biocut Microtome (Leica, Wetzlar, Germany) at a thickness of $3 \mu \mathrm{m}$, and stained with hematoxylin-eosin. Assay of incorporation of bromodeoxyuridine (BrdU, Sigma) was performed as described [17]. Apoptotic cells were detected with the TUNEL (terminal deoxynucleotidyl transferase-mediated dUTP-biotin nick end labeling) assay as previously described [19]. 


\section{Results}

\subsection{Generation of mice lacking all Cip/Kip CKIs}

To examine the requirement for Cip/Kip CKIs during embryonic development, we intercrossed $p 21^{-/-} p 27^{+/-} p 57^{[+] /+}$males with $p 21^{+/-} p 27^{+/-} p 57^{[-] /+}$females, or $p 21^{+/-} p 27^{+/-}$ $p 57^{[+] /+}$males with $p 21^{-/-} p 27^{+-} p 57^{[-] /+}$females (with the symbol in brackets denoting the paternally derived p57 allele, which is transcriptionally repressed by genomic imprinting) [5, 6, 9]. We focused our analysis on $p 21^{+/-} p 27^{+/+} p 57^{[+] /+}$(control), $p 21^{-/-} p 27^{1-} p 57^{[+] /+}$(p21/p27 DKO), $p 21^{-1-} p 27^{+/+} p 57^{[+] /-}$(p21/p57 DKO), $p 21^{+/-} p 27^{1-} p 57^{[+] /-}(\mathrm{p} 27 / \mathrm{p} 57 \mathrm{DKO})$, and $p 21^{-/-}$ $p 27^{-1} p 57^{[+] /-}$(TKO) mice. Given that all p27/p57 DKO mice die in utero by E16.5, we examined the genotypes of embryos generated by the intercrosses at E11.5, E13.5, and E15.5. There was no or only a slight decrease in the survival of control, p21/p27 DKO, or p21/p57 DKO mice through E15.5, whereas two-thirds of p27/p57 DKO and all TKO embryos had died by E15.5 (Figure 1A). The difference in survival at E15.5 between p27/p57 DKO and TKO embryos may be attributable to a compensatory role of p21. Unexpectedly, however, $>70 \%$ of TKO embryos survived until E13.5, when most tissues and organs are already formed (Figure 1A and B). At E15.5, most TKO mice exhibited subcutaneous hemorrhage and pericardial edema (Figure 1B), although the precise cause of death was unclear from gross inspection. These results suggested that Cip/Kip CKIs are not required until midgestation.

We examined the expression of Cip/Kip CKIs at the mRNA and protein levels by RT-PCR analysis (Figure 1C) and immunoblot analysis (Figure 1D), respectively, in MEFs prepared from control, p21/p27 DKO, p21/p57 DKO, p27/p57 DKO, and TKO mice. Compensatory up-regulation of the remaining Cip/Kip CKI in DKO MEFs was not observed at either the mRNA or protein level. We confirmed that no Cip/Kip CKI mRNA or protein was expressed in TKO MEFs.

\subsection{Placental defects in Cip/Kip-null embryos}

We investigated the reason for the mortality of TKO embryos by microscopic inspection. Given that p57 KO [11, 12, 17], p21/p57 DKO [15], and p27/p57 DKO [16] embryos all manifest a developmental defect of the placenta, we examined the placenta of 
control, p27/p57 DKO, and TKO embryos by hematoxylin-eosin staining (Figure 2A). Deposition of fibrin in the labyrinth zone was apparent for both p27/p57 DKO and TKO embryos as early as E11.5. Massive cell death was also evident in the same region, likely as a result of blockade of the blood supply caused by fibrin deposition. We measured the rates of cell proliferation and apoptosis in the labyrinth zone of the placenta with BrdU incorporation and TUNEL assays, respectively. As shown previously [16], the placenta of p27/p57 DKO embryos exhibited an increase in the rate of cell proliferation compared with that of control embryos (Figure 2B). The placenta of TKO embryos also manifested an increased rate of cell proliferation, although the rate did not differ significantly from that in the placenta of p27/p57 DKO embryos. Similarly, the frequency of TUNEL-positive (apoptotic) cells was markedly greater in the placenta of p27/p57 DKO or TKO embryos than in that of control embryos (Figure 2C); again, the frequency of apoptosis did not differ significantly between p27/p57 DKO and TKO embryos, although that in the latter tended to be higher than that in the former. We also examined other organs of the embryos, including heart, lung, liver, intestine, kidney, and skeletal muscle. Hematoxylin-eosin staining as well as BrdU incorporation and TUNEL assays did not reveal any obvious defects with the exception of pericardial effusion, which was probably associated with the placental abnormalities, in p27/p57 DKO and TKO embryos (data not shown).

\subsection{Lens defects in Cip/Kip-null embryos}

We next investigated ocular lens development. In control embryos at E11.5, cells in the posterior region of the lens exit the cell cycle and begin to elongate in the direction of the anterior wall (Figure 3A). These cells then proliferate and migrate to the equatorial zone, where they express high levels of p57 and differentiate to form secondary lens fiber cells [16, 20, 21]. Lens cells deficient in p57 proliferate for a longer period than do control cells, but they eventually undergo cell cycle exit and differentiate into lens fiber cells, suggesting the existence of a second regulatory pathway (in addition to that mediated by p57) for the control of cell cycle exit in this tissue [16]. Defects in lens development were not previously observed in p27-deficient mice [7-9]. Whereas cell elongation is complete and the differentiated fiber cells are capped at the anterior wall by a layer of immature epithelial cells in control embryos at E13.5, nuclei remained located in the posterior region of the lens in 
both p27/p57 DKO and TKO embryos at this stage (Figure 3A). Large vacuoles were also apparent in the anterior region of the lens in both p27/p57 DKO and TKO embryos, likely as a result of the failure of fiber cells to elongate or of cell death by both apoptosis and necrosis [16].

The BrdU incorporation (Figure 3B) and TUNEL (Figure 3C) assays revealed that the numbers of proliferating and apoptotic cells in the lens were increased to similar extents in both p27/p57 DKO and TKO embryos compared with control embryos. These results thus suggested that, among Cip/Kip CKIs, p27 and p57 are largely responsible for regulation of the cell cycle and differentiation in lens cells, whereas p21 does not influence cell proliferation or apoptosis during lens development.

\subsection{Role of Cip/Kip CKIs in cell cycle progression in MEFs}

We investigated the effect of Cip/Kip CKI loss on the cell cycle in MEFs prepared from control, p21/p27 DKO, p21/p57 DKO, p27/p57 DKO, and TKO embryos. The rate of cell proliferation did not differ among these genotypes (Figure 4A). Flow cytometric analysis also revealed that the lack of any two or all three Cip/Kip CKIs did not significantly affect the proportions of cells in $\mathrm{G}_{0}-\mathrm{G}_{1}, \mathrm{~S}$, or $\mathrm{G}_{2}-\mathrm{M}$ phases of the cell cycle (Figure $4 \mathrm{~B}$ ). Neither the kinase activity associated with CDK1, CDK2, or CDK4 nor the abundance of CDKs or cyclins in asynchronous MEFs differed among the genotypes tested, with the exception that the amount of cyclin D1 was reduced in all DKO and TKO MEFs compared with control cells (Figure 4C and D).

We also examined progression of the cell cycle from $G_{0}$ to $S$ phase. MEFs that had been restrained in $\mathrm{G}_{0}$ phase by serum deprivation were released by serum replenishment, and the percentage of cells in $S$ phase was monitored (Figure 4E). The timing of entry into $S$ phase did not differ among MEF genotypes. The kinase activity associated with CDK2 or CDK4 as well as the levels of CDKs and cyclins were also examined (Figure 4F). The abundance and kinase activity of $\mathrm{CDK} 2$ during early progression from $\mathrm{G}_{0}$ to $\mathrm{S}$ phase were markedly increased in both p27/p57 DKO and TKO MEFs compared with control cells. CDK2 activity was also greater in TKO MEFs than in p27/p57 DKO MEFs at 12 and $16 \mathrm{~h}$ after serum replenishment. The expression of cyclin $A$, which is normally undetectable in $G_{0}$ phase, was increased in p27/p57 DKO and TKO MEFs in this phase. On the other hand, the 
kinase activity associated with CDK4 was slightly decreased in both p27/p57 DKO and TKO MEFs in the early period after serum replenishment, likely as a result of the observed decrease in the amount of cyclin D1. The extent of the reduction in CDK4 activity and the amount of cyclin D1 was greater in TKO MEFs than in p27/p57 DKO MEFs. Collectively, these results suggested that formation of the CDK4-cyclin D1 complex is dependent in part on the presence of all three Cip/Kip CKIs, even though these CKIs are dispensable for normal progression of the cell cycle. 


\section{Discussion}

Cip/Kip CKIs share a conserved $\mathrm{NH}_{2}$-terminal domain and function to inhibit CDKs (especially CDK2) as well as to promote the association of D-type cyclins with CDK4. Mice lacking one or any two of the three Cip/Kip CKIs have been generated and characterized in detail [6-16]. Mice deficient in a molecule that plays a key role in development often show a compensatory increase in the expression of other molecules with similar functions. Such compensation is thought to ameliorate the phenotype of the mutant mice, and, in the case of Cip/Kip mutant mice, may mask the essential function of these CKIs during development. We thus generated mice lacking all three Cip/Kip CKIs in order to evaluate the effect of the complete loss of these negative regulators of the cell cycle.

The resulting TKO mice were unexpectedly found to survive until midgestation. The death of TKO embryos appears to occur slightly earlier than that of p27/p57 DKO mice, which exhibit the most severe phenotype among the three types of DKO mice. These results suggest that p21 might contribute to the improved survival of p27/p57 DKO mice compared with that of TKO mice, although p27 and p57 are integral to mouse development around the midgestation period. We did not detect any compensatory increase in the expression of the remaining CKI in DKO MEFs, whereas such an increase has been detected in some adult tissues of DKO mice [4]. This difference might be due to the fact that the MEFs were prepared from embryos at E13.5, when most DKO mice are still alive and Cip/Kip CKIs are not essential for cell cycle regulation. A compensatory up-regulation of the remaining Cip/Kip CKI may thus not confer a selective advantage in MEFs. Consistent with this notion, we did not observe any difference in growth rate between DKO or TKO MEFs and control MEFs.

Microscopic examination of embryonic tissues, especially the placenta and lens, revealed that the defects in TKO mice did not differ substantially from those in p27/p57 DKO mice. Both p27 and p57 appear to be essential, and p21 dispensable, for normal development of these tissues. The structures of p27 and p57 are more similar to each other than to that of p21, and these two CKIs have overlapping functions in development. Indeed, we have shown that p27 and p57 are functionally interchangeable by generation of knock-in mice $[4,17]$. The differences between p27 and p57 KO mice may therefore be attributable not to a difference in 
molecular function between these two proteins but to differences in their spatiotemporal patterns of expression. Whereas p27 is expressed mostly in adult tissues, p57 is widely expressed in embryonic tissues, and, with the exception of stem cells, in which p57 dominates p21 and p27 [4], its expression ceases after birth [22]. Given that p21 operates predominantly under the control of p53, it might serve as an "emergency brake" of the cell cycle that is triggered by exposure of cells to genotoxic insults. This notion is consistent with the observation that mice deficient in p21 or p53 are viable and do not show any gross abnormalities during embryonic development. 


\section{Acknowledgments}

We thank P. Leder for p21-deficient mice; M. Tanaka, N. Nishimura, and N. Kinoshita for technical assistance; K. Yumimoto and other members of our laboratories for discussion; and A. Ohta for help in preparation of the manuscript. This study was supported in part by a Grant-in-Aid from the Ministry of Education, Culture, Sports, Science, and Technology of Japan and by the Global Center of Excellence Program of the Japanese government. 


\section{References}

[1] C.J. Sherr, J.M. Roberts, CDK inhibitors: positive and negative regulators of G1-phase progression. Genes Dev. 13 (1999) 1501-1512.

[2] J. LaBaer, M.D. Garrett, L.F. Stevenson, J.M. Slingerland, C. Sandhu, H.S. Chou, A. Fattaey, E. Harlow, New functional activities for the p21 family of CDK inhibitors. Genes Dev. 11 (1997) 847-862.

[3] K. Nakayama, K.I. Nakayama, Cip/Kip cyclin-dependent kinase inhibitors: brakes of the cell cycle engine during development. Bioessays 20 (1998) 1020-1029.

[4] A. Matsumoto, S. Takeishi, T. Kanie, E. Susaki, I. Onoyama, Y. Tateishi, K. Nakayama, K.I. Nakayama, p57 is required for quiescence and maintenance of adult hematopoietic stem cells. Cell Stem Cell 9 (2011) 262-271.

[5] I. Hatada, T. Mukai, Genomic imprinting of $\mathrm{p} 57^{\mathrm{KIP} 2}$, a cyclin-dependent kinase inhibitor, in mouse. Nat. Genet. 11 (1995) 204-206.

[6] C. Deng, P. Zhang, J.W. Harper, S.J. Elledge, P. Leder, Mice lacking p $21^{\text {CIP1/WAF1 }}$ undergo normal development, but are defective in G1 checkpoint control. Cell 82 (1995) 675-684.

[7] M.L. Fero, M. Rivkin, M. Tasch, P. Porter, C.E. Carow, E. Firpo, K. Polyak, L.H. Tsai, V. Broudy, R.M. Perlmutter, K. Kaushansky, J.M. Roberts, A syndrome of multiorgan hyperplasia with features of gigantism, tumorigenesis, and female sterility in p $27^{\text {Kip } 1}$. deficient mice. Cell 85 (1996) 733-744.

[8] H. Kiyokawa, R.D. Kineman, K.O. Manova-Todorova, V.C. Soares, E.S. Hoffman, M. Ono, D. Khanam, A.C. Hayday, L.A. Frohman, A. Koff, Enhanced growth of mice lacking the cyclin-dependent kinase inhibitor function of p2 ${ }^{\text {Kipl }}$. Cell 85 (1996) 721732.

[9] K. Nakayama, N. Ishida, M. Shirane, A. Inomata, T. Inoue, N. Shishido, I. Horii, D.Y. Loh, K.I. Nakayama, Mice lacking p $27^{\text {Kipl }}$ display increased body size, multiple organ hyperplasia, retinal dysplasia, and pituitary tumors. Cell 85 (1996) 707-720.

[10] K. Takahashi, K. Nakayama, Mice lacking a CDK inhibitor, p57Kip2, exhibit skeletal abnormalities and growth retardation. J. Biochem. 127 (2000) 73-83.

[11] Y. Yan, J. Frisen, M.H. Lee, J. Massague, M. Barbacid, Ablation of the CDK inhibitor p5 $7^{\text {Kip2 }}$ results in increased apoptosis and delayed differentiation during mouse development. Genes Dev. 11 (1997) 973-983.

[12] P. Zhang, N.J. Liegeois, C. Wong, M. Finegold, H. Hou, J.C. Thompson, A. Silverman, J.W. Harper, R.A. DePinho, S.J. Elledge, Altered cell differentiation and proliferation in mice lacking $\mathrm{p} 57^{K I P 2}$ indicates a role in Beckwith-Wiedemann syndrome. Nature 387 (1997) 151-158.

[13] U. Sankar, K. Patel, T.J. Rosol, M.C. Ostrowski, RANKL coordinates cell cycle withdrawal and differentiation in osteoclasts through the cyclin-dependent kinase inhibitors $\mathrm{p} 27^{\mathrm{KIP} 1}$ and p21 ${ }^{\mathrm{CIP} 1}$. J. Bone Miner. Res. 19 (2004) 1339-1348.

[14] M. Mukai, Q. Dong, M.P. Hardy, H. Kiyokawa, R.E. Peterson, P.S. Cooke, Altered prostatic epithelial proliferation and apoptosis, prostatic development, and serum testosterone in mice lacking cyclin-dependent kinase inhibitors. Biol. Reprod. 73 (2005) 951-958.

[15] P. Zhang, C. Wong, D. Liu, M. Finegold, J.W. Harper, S.J. Elledge, p2 ${ }^{\mathrm{CIP} 1}$ and p57 ${ }^{\mathrm{KIP} 2}$ 
control muscle differentiation at the myogenin step. Genes Dev. 13 (1999) 213-224.

[16] P. Zhang, C. Wong, R.A. DePinho, J.W. Harper, S.J. Elledge, Cooperation between the Cdk inhibitors $\mathrm{p} 27^{\mathrm{KIP} 1}$ and $\mathrm{p} 57^{\mathrm{KIP} 2}$ in the control of tissue growth and development. Genes Dev. 12 (1998) 3162-3167.

[17] E. Susaki, K. Nakayama, L. Yamasaki, K.I. Nakayama, Common and specific roles of the related CDK inhibitors p27 and p57 revealed by a knock-in mouse model. Proc. Natl. Acad. Sci. U. S. A. 106 (2009) 5192-5197.

[18] N. Ishida, M. Kitagawa, S. Hatakeyama, K.I. Nakayama, Phosphorylation at serine 10, a major phosphorylation site of $\mathrm{p} 27^{\text {Kipl }}$, increases its protein stability. J. Biol. Chem. 275 (2000) 25146-25154.

[19] T. Moroishi, M. Nishiyama, Y. Takeda, K. Iwai, K.I. Nakayama, The FBXL5-IRP2 axis is integral to control of iron metabolism in vivo. Cell Metab. 14 (2011) 339-351.

[20] J.W. McAvoy, Induction of the eye lens. Differentiation 17 (1980) 137-149.

[21] J. Piatigorsky, Lens differentiation in vertebrates. A review of cellular and molecular features. Differentiation 19 (1981) 134-153.

[22] H. Nagahama, S. Hatakeyama, K. Nakayama, M. Nagata, K. Tomita, K.I. Nakayama, Spatial and temporal expression patterns of the cyclin-dependent kinase (CDK) inhibitors p $27^{\text {Kip } 1}$ and p $57^{\text {Kip } 2}$ during mouse development. Anat. Embryol. 203 (2001) 77-87. 


\section{Footnotes}

${ }^{1}$ Abbreviations: CDK, cyclin-dependent kinase; CKI, CDK inhibitor; E, embryonic day; MEF, mouse embryonic fibroblast; RT, reverse transcription; PCR, polymerase chain reaction; ARBP, acidic ribosomal phosphoprotein; BrdU, bromodeoxyuridine; TUNEL, terminal deoxynucleotidyl transferase-mediated dUTP-biotin nick end labeling. 


\section{Figure Legends}

Fig. 1. Mice deficient in all Cip/Kip family CKIs die earlier than those deficient in both p27 and p57. (A) Summary of genotypes determined for embryos generated from intercrosses of $p 21^{-/-} p 27^{+/-} p 57^{[+] /+}$males with $p 21^{+/-} p 27^{+/-} p 57^{[-] /+}$females, or of $p 21^{+/-} p 27^{+/-} p 57^{[+] /+}$males with $p 21^{-/-} p 27^{+/-} p 57^{[-] /+}$females. Observed/expected numbers for each genotype are shown at E11.5, E13.5, and E15.5, with "observed" referring to the number of live embryos at the time of inspection; the total number of live and dead embryos of each genotype is indicated in parentheses. Survival rate was calculated as live/total embryos at each stage. (B) Representative appearance of E11.5, E13.5, and E15.5 embryos of the indicted genotypes. Scale bars, $5 \mathrm{~mm}$. (C) RT-PCR analysis of p21, p27, p57, and ARBP (control) mRNAs in MEFs derived from embryos of the indicated genotypes. (D) Immunoblot (IB) analysis of p21, p27, p57, and heat shock protein 90 (HSP90, control) in the indicated MEF lines after incubation with the proteasome inhibitor MG132 $(10 \mu \mathrm{M})$ for $8 \mathrm{~h}$. Asterisk indicates nonspecific bands.

Fig. 2. Placental abnormalities of mice deficient in all Cip/Kip family members. (A) Hematoxylin-eosin staining of the placenta for mice of the indicated genotypes at various developmental stages. Arrows indicate necrosis in the embryo-derived labyrinth zone. Scale bars, $500 \mu \mathrm{m}$. (B) In situ BrdU incorporation in the placental labyrinth zone was examined for mice of the indicated genotypes at E13.5 (left); scale bar, $100 \mu \mathrm{m}$. The percentage of BrdU-positive cells in such images was quantified (right); data are means \pm SD of values obtained from five placentas of each genotype. ${ }^{*} P<0.05$, $* * P<0.01$; n.s., not significant. (C) TUNEL staining of the placental labyrinth zone was examined for mice of the indicated genotypes at E13.5 (left); scale bar, $100 \mu \mathrm{m}$. The number of TUNEL-positive cells in such images was also quantified (right); data are means \pm SD of values obtained from six placentas of each genotype. $* * P<0.01$.

Fig. 3. Lens abnormalities of mice deficient in all Cip/Kip family members are similar to those of p27/p57 DKO mice. (A) Hematoxylin-eosin staining of the lens from mice of the indicated genotypes at various developmental stages. Arrows indicate nuclei at the posterior 
edge of the lens. Scale bars, $100 \mu \mathrm{m}$. (B) In situ BrdU incorporation in the lens was examined for mice of the indicated genotypes at E13.5 (left). Arrows indicate dividing cells at the posterior edge of the lens. Scale bar, $100 \mu \mathrm{m}$. The percentage of BrdU-positive cells in such images was quantified (right); data are means \pm SD of values obtained from five or six lenses of each genotype. ${ }^{* *} P<0.01$. (C) TUNEL staining of the lens from mice of the indicated genotypes at E13.5 (left); scale bar, $100 \mu \mathrm{m}$. The number of TUNEL-positive cells in such images was determined (right); data are means \pm SD of values obtained from six lenses of each genotype. $* P<0.05, * * P<0.01$.

Fig. 4. Cell cycle progression in MEFs deficient in all Cip/Kip family members. (A) Growth curves for MEFs of the indicated genotypes. Data are means \pm SD of values obtained with three MEF preparations of each genotype. (B) Cell cycle distribution for asynchronous MEFs as determined by flow cytometry. Data are means \pm SD of values obtained with three MEF preparations of each genotype. (C) In vitro kinase assays for immunoprecipitated (IP) CDK1, CDK2, and CDK4 with histone $\mathrm{H} 1$ (for CDK1 and CDK2) or GST-Rb-C (for CDK4) as substrate. (D) Immunoblot analysis of CDKs and cyclins in asynchronous MEFs. (E) Kinetics of the reentry of MEFs into the cell cycle from $G_{0}$ phase. Data are means \pm SD of values obtained with three MEF preparations of each genotype. (F) In vitro kinase assays for immunoprecipitated CDK2 and CDK4 as well as immunoblot analysis of CDKs and cyclins for MEFs released from $\mathrm{G}_{0}$ arrest for the indicated times. 


\begin{tabular}{|c|c|c|c|c|c|c|}
\hline \multirow[b]{2}{*}{ Stage } & \multicolumn{5}{|c|}{$\begin{array}{c}\text { Observed (total)/expected } \\
\text { Survival rate }\end{array}$} & \multirow[b]{2}{*}{ Total } \\
\hline & $\begin{array}{l}p 21^{+/-} \\
p 27^{+/+} \\
p 57^{[+] /+}\end{array}$ & $\begin{array}{l}p 21^{-/-} \\
p 27^{-/-} \\
p 57^{[+] /+}\end{array}$ & $\begin{array}{l}p 21^{-/-} \\
p 27^{+/+} \\
p 57^{[+] /-}\end{array}$ & $\begin{array}{l}p 21^{+/-} \\
p 27^{-/-} \\
p 57^{[+] /-}\end{array}$ & $\begin{array}{l}p 21^{-/-} \\
p 27^{-/-} \\
p 57^{[+] /-}\end{array}$ & \\
\hline E11.5 & $\begin{array}{c}28(29) / 18.5 \\
97 \%\end{array}$ & $\begin{array}{c}19(20) / 18.5 \\
95 \%\end{array}$ & $\begin{array}{c}17(17) / 18.5 \\
100 \%\end{array}$ & $\begin{array}{c}8(9) / 18.5 \\
89 \%\end{array}$ & $\begin{array}{c}16(20) / 18.5 \\
80 \%\end{array}$ & 296 \\
\hline E13.5 & $\begin{array}{c}15(15) / 17.5 \\
100 \%\end{array}$ & $\begin{array}{c}19(20) / 17.5 \\
95 \%\end{array}$ & $\begin{array}{c}15(17) / 17.5 \\
88 \%\end{array}$ & $\begin{array}{c}11(20) / 17.5 \\
55 \%\end{array}$ & $\begin{array}{c}15(21) / 17.5 \\
71 \%\end{array}$ & 280 \\
\hline E15.5 & $\begin{array}{c}15(15) / 17.3 \\
100 \%\end{array}$ & $\begin{array}{c}14(17) / 17.3 \\
82 \%\end{array}$ & $\begin{array}{c}17(21) / 17.3 \\
81 \%\end{array}$ & $\begin{array}{c}3(9) / 17.3 \\
33 \%\end{array}$ & $\begin{array}{c}0(20) / 17.3 \\
0 \%\end{array}$ & 277 \\
\hline
\end{tabular}

B

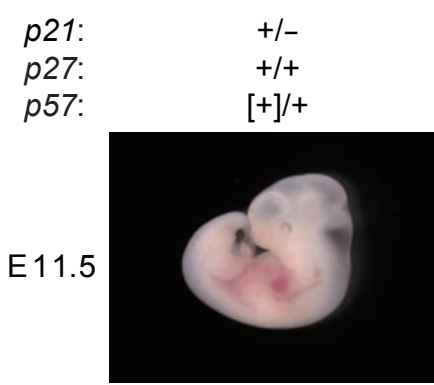

$+/-$

$[+] /-$

$-1-$

$[+] /-$

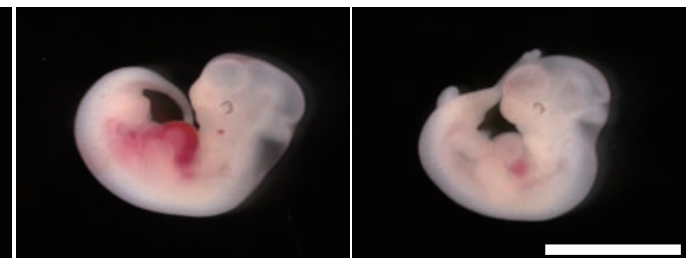

E13.5
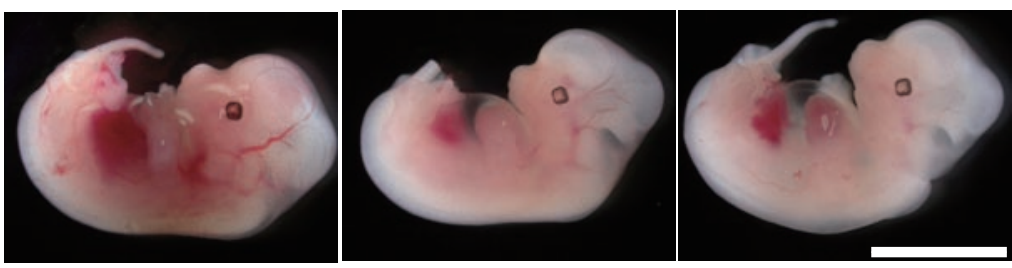

E15.5
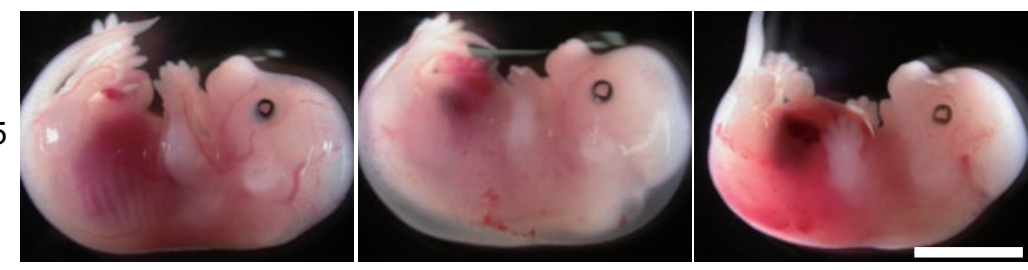

C

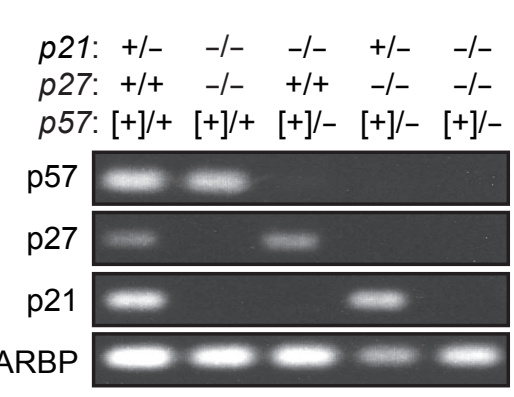

D

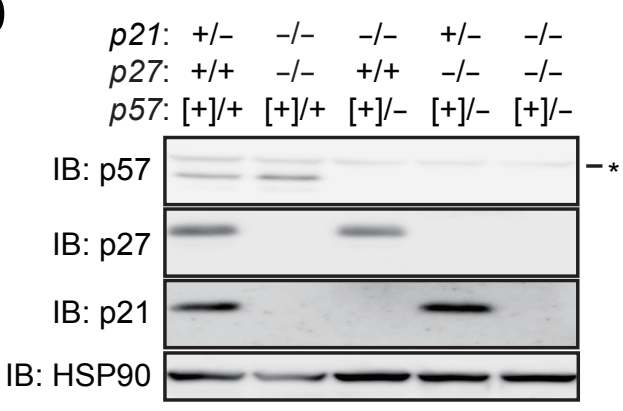

Tateishi et al. Figure 1 
A

$p 21$
$p 27$

+ -

p57:

$[+] /+$

+-
$-/-$

$[+] /-$

$-1-$
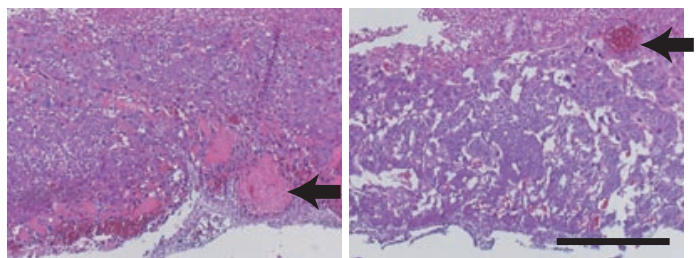

E13.5
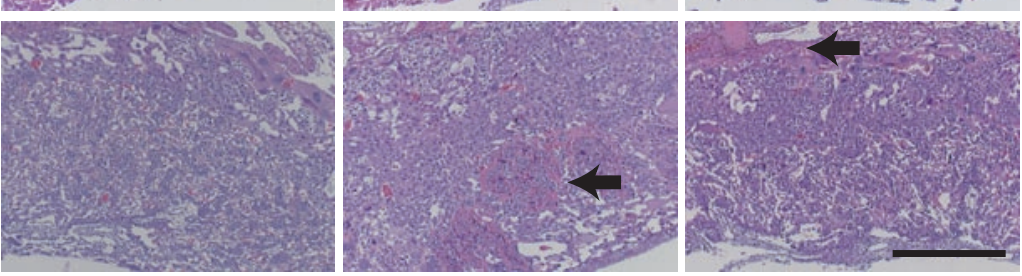

E15.5
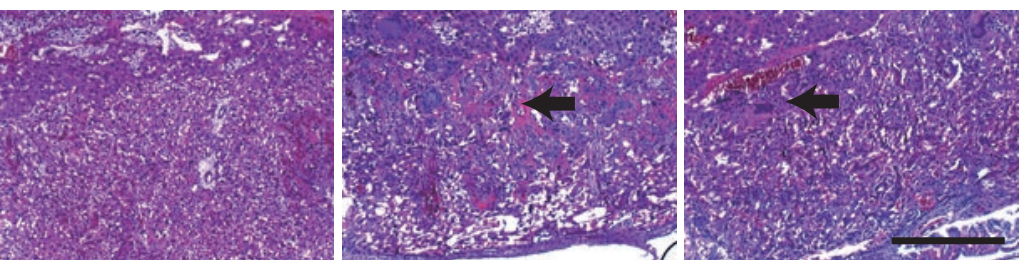

B
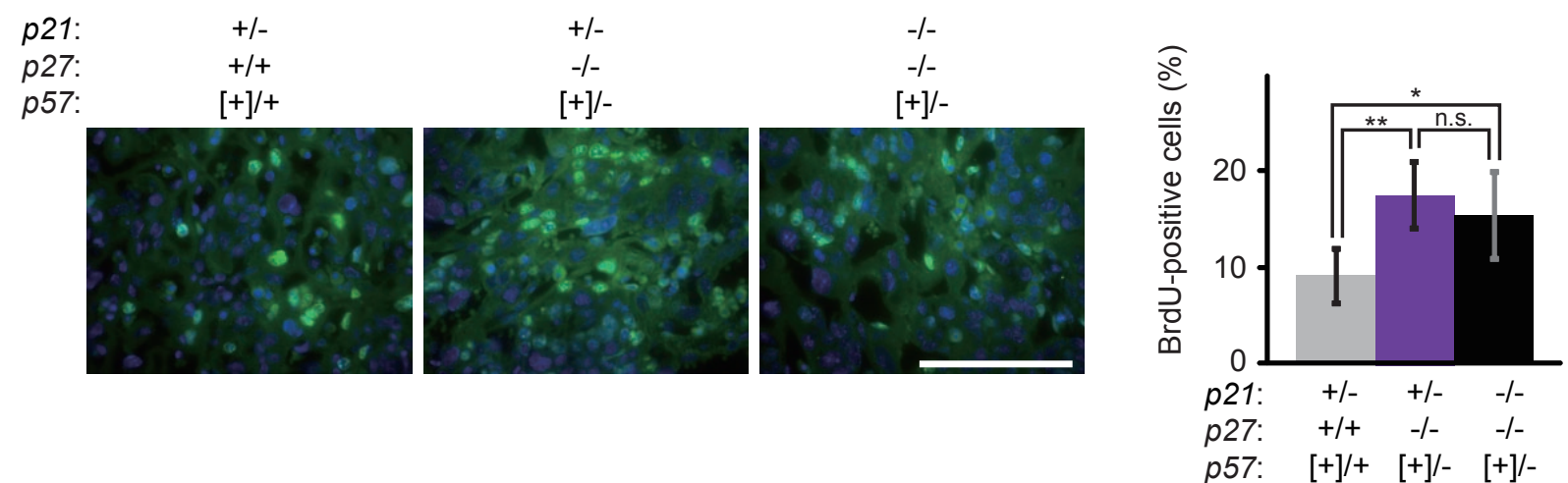

C
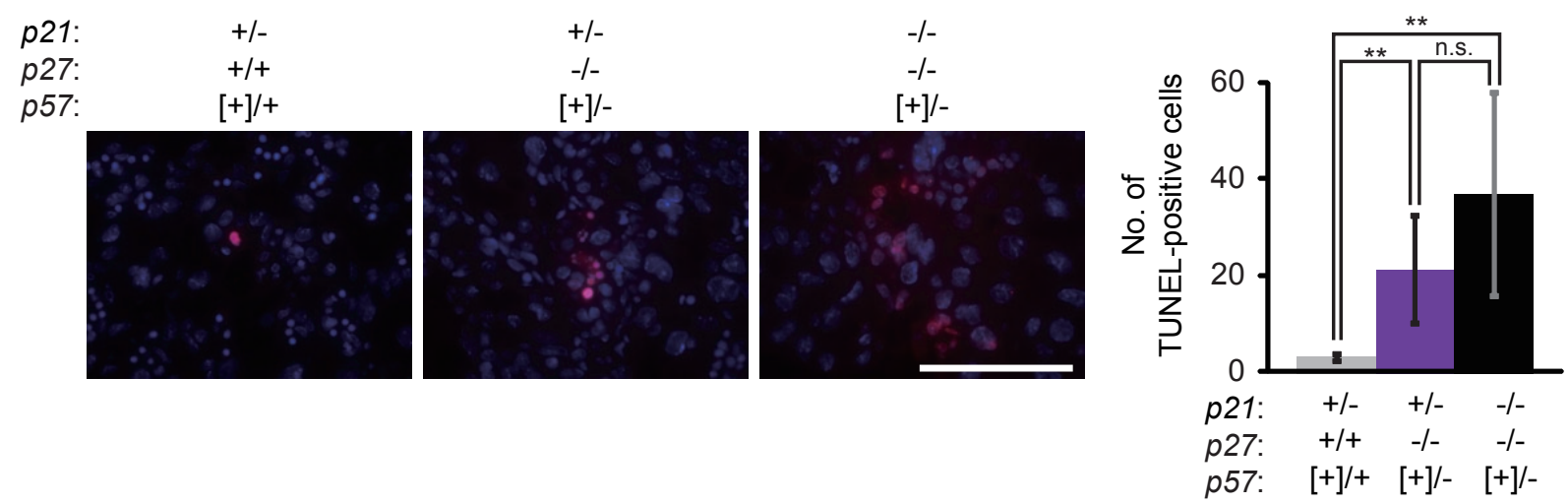

Tateishi et al. Figure 2 
A

$\begin{array}{ll}\text { p21: } & +/- \\ \text { p27: } & +/+ \\ \text { p57: } & {[+] /+}\end{array}$

E11.5

E13.5

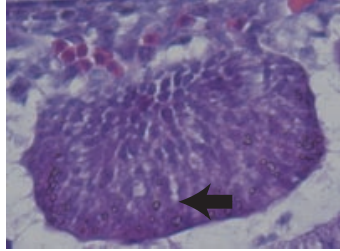

E15.5
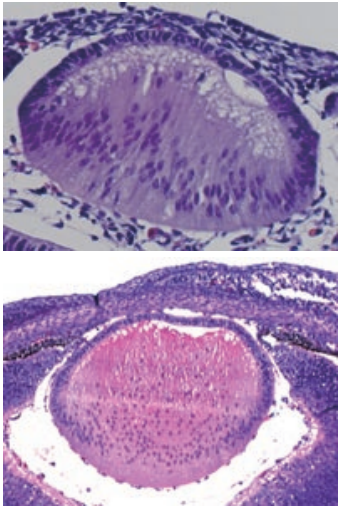

B
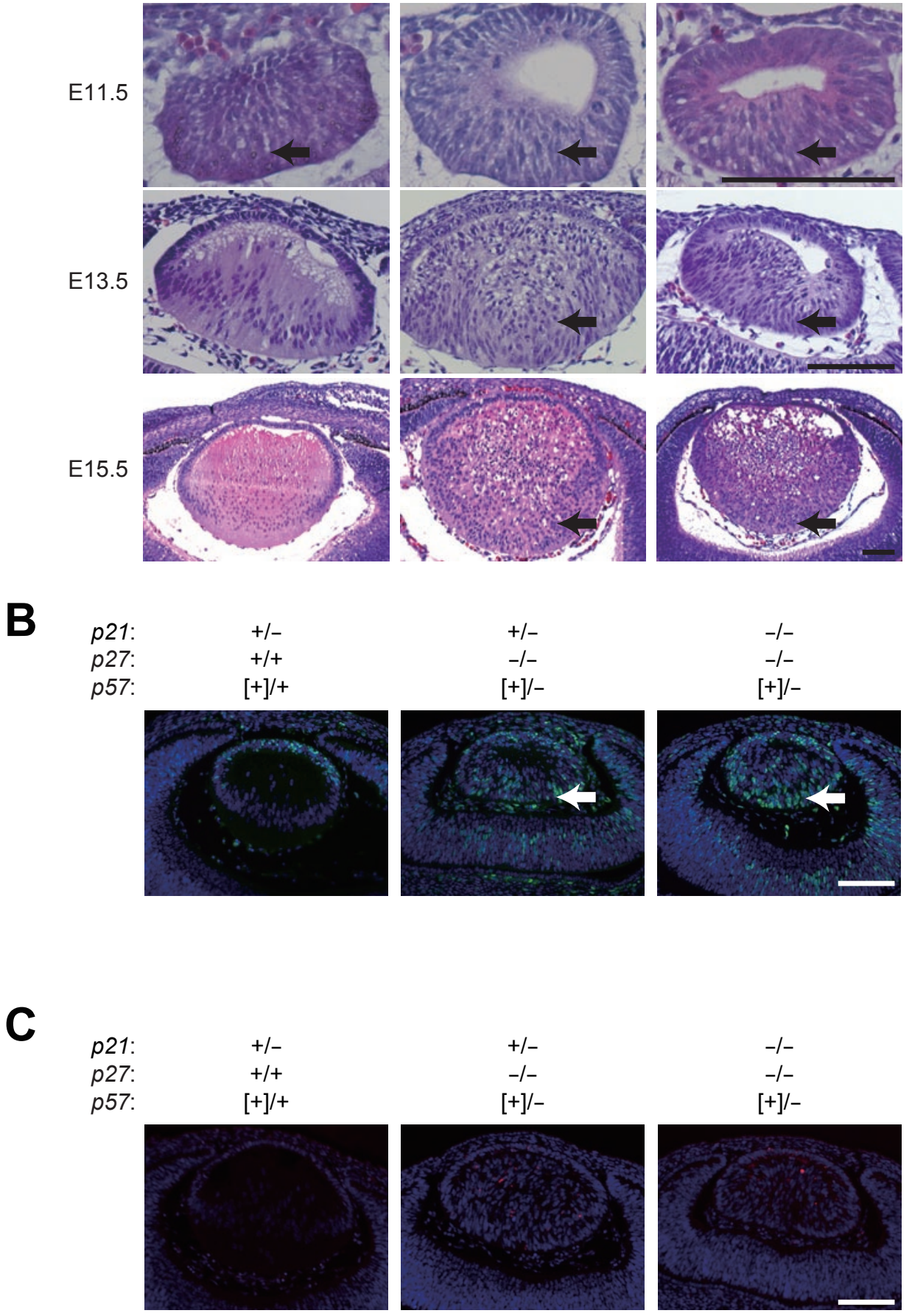

B

C

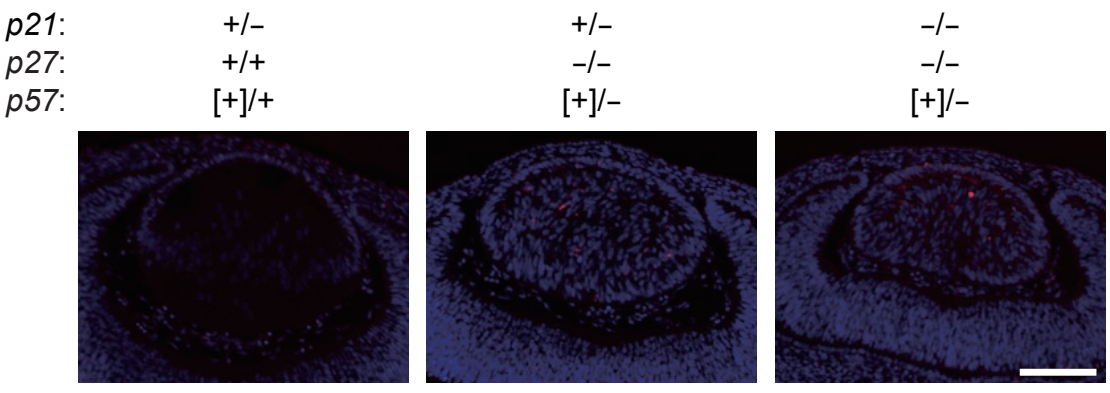

Tateishi et al. Figure 3
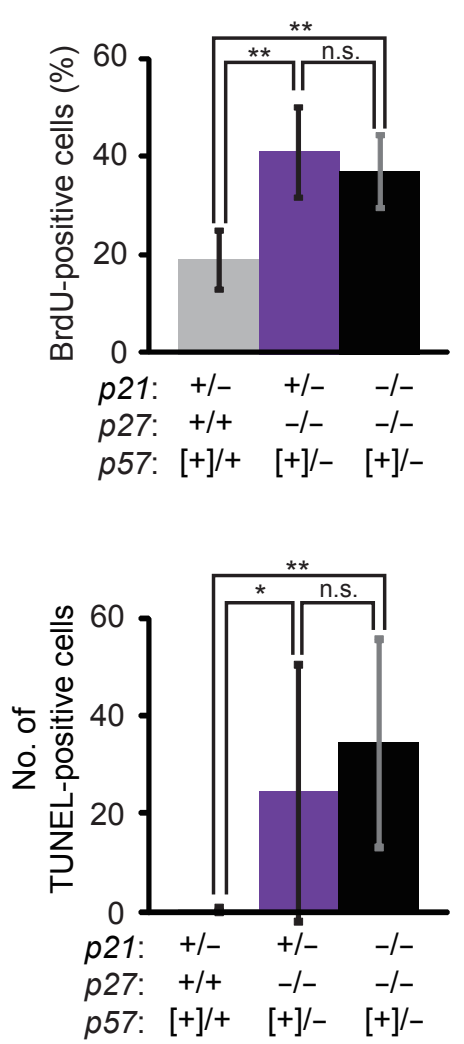
A

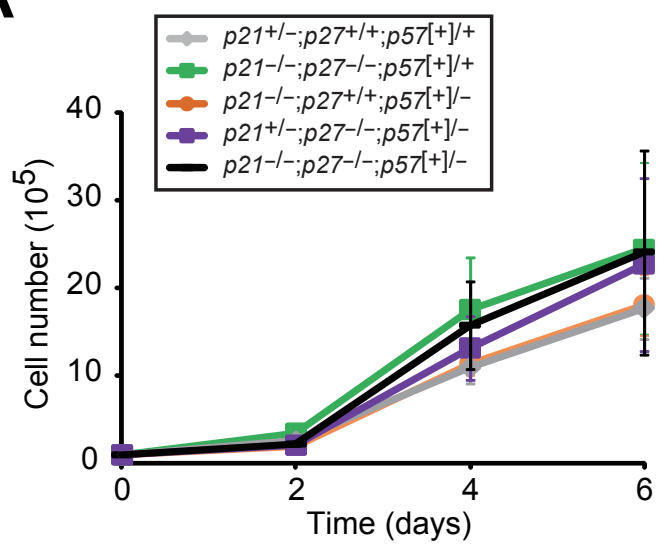

C

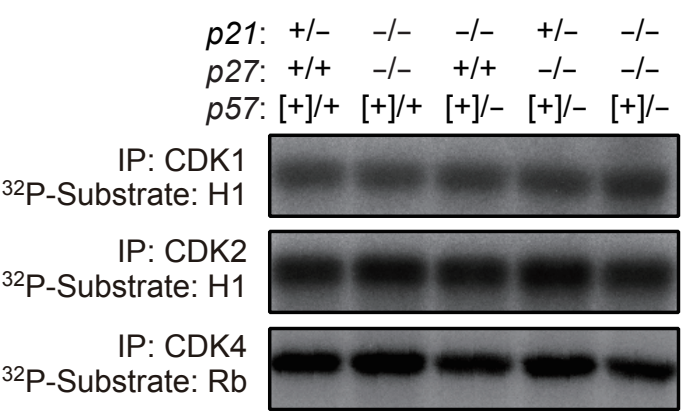

E

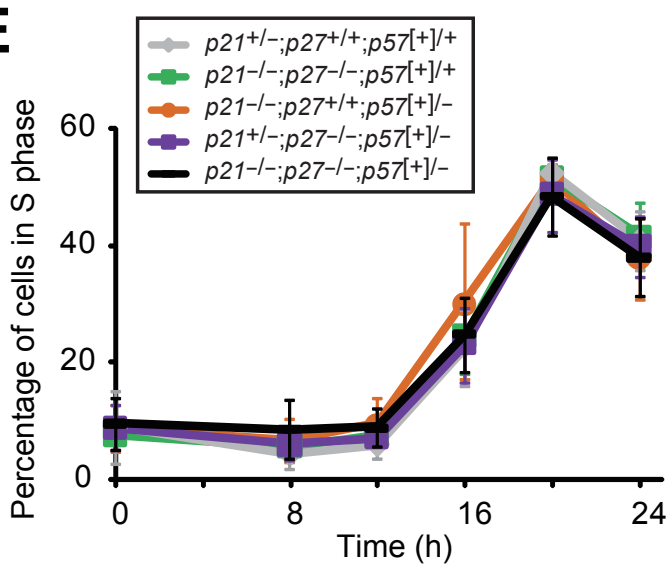

B

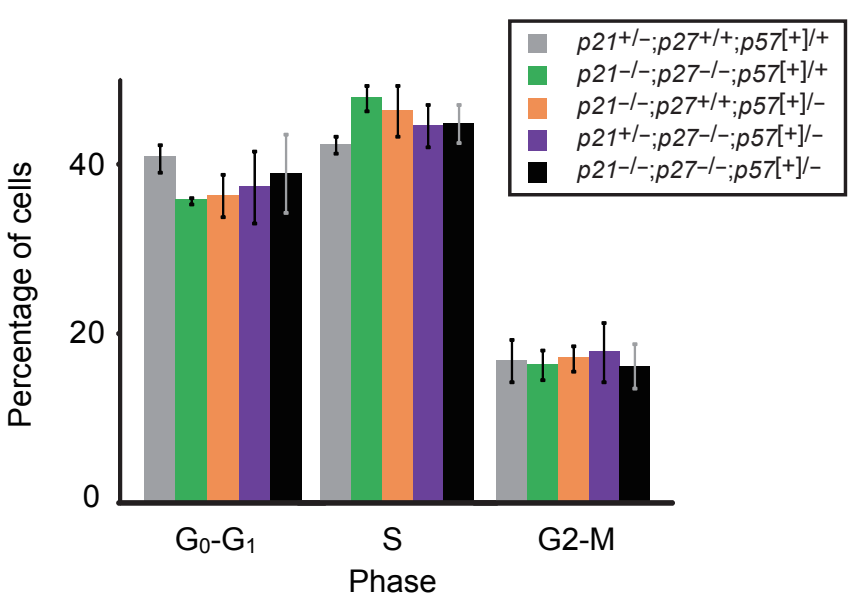

D $\begin{array}{llllll}\text { p21: }+/- & -/- & -/- & +/- & -/- \\ \text { p27: }+/+ & -/- & +/+ & -/- & -/- \\ \text { p57: }[+] /+ & {[+] /+} & {[+] /-} & {[+] /-} & {[+] /-}\end{array}$

IB: CDK1

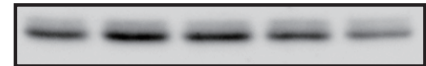

IB: CDK2

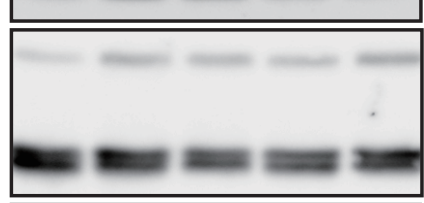

IB: CDK4

IB: Cyclin B1

IB: Cyclin A

IB: Cyclin D1

IB: HSP90
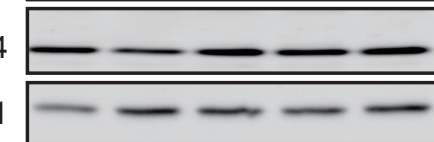

A
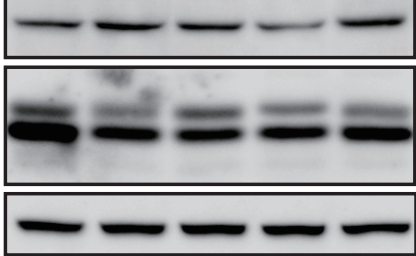

F Time after $\mathrm{G}_{0}$ release $(\mathrm{h})$ :

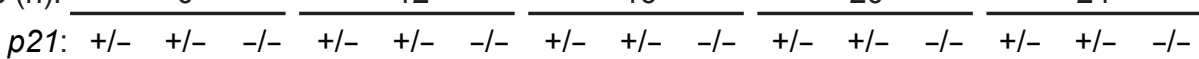

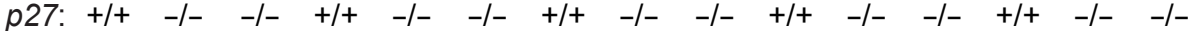
p57: $[+] /+[+] /-[+] /-[+] /+[+] /-[+] /-[+] /+[+] /-[+] /-[+] /+[+] /-[+] /-[+] /+[+] /-[+] /-$ IP: CDK2 ${ }^{32} \mathrm{P}$-Substrate: $\mathrm{H} 1$ IP: CDK4 ${ }^{32} \mathrm{P}$-Substrate: $\mathrm{Rb}$

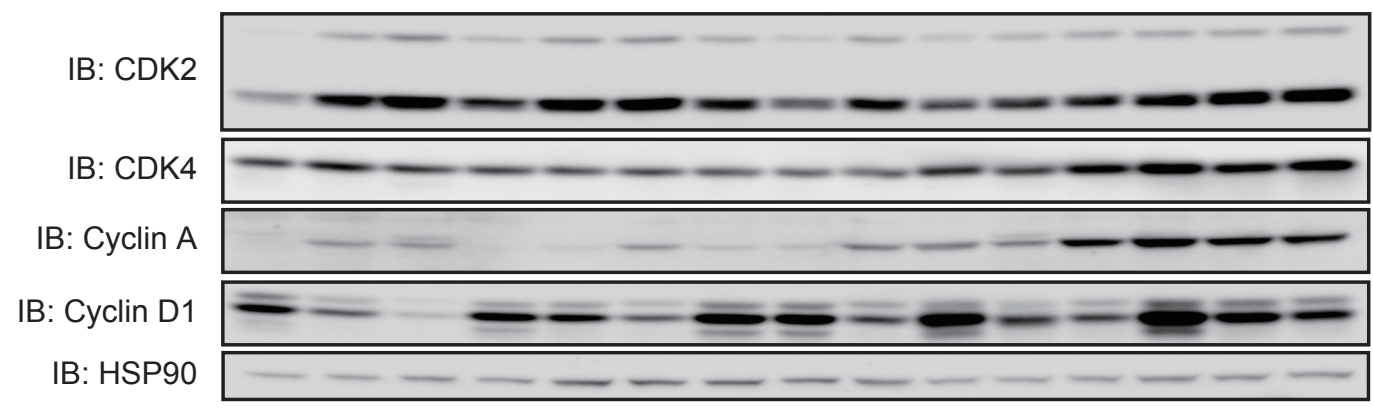

